\title{
Nuclear medium effects from hadronic atoms
}

\author{
E. Friedman, A. Gal \\ Racah Institute of Physics, The Hebrew University, Jerusalem 91904, Israel \\ E-mail: elifried@vms.huji.ac.il, avragal@vms.huji.ac.il
}

\begin{abstract}
The state of the art in the study of $\pi^{-}, K^{-}$and $\Sigma^{-}$atoms, along with the inmedium nuclear interactions deduced for these hadrons, is reviewed. A special emphasis is placed on recent developments in $\bar{K}$-nuclear physics, where a strongly attractive density dependent $K^{-}$-nuclear potential of order $150-200 \mathrm{MeV}$ in nuclear matter emerges by fitting $\mathrm{K}^{-}$-atom data. This has interesting repercussions on $\bar{K}$ quasibound nuclear states, on the composition of strange hadronic matter and on $\bar{K}$ condensation in self bound hadronic systems.
\end{abstract}

\section{Introduction}

Hadronic atoms have played an important role in elucidating in-medium properties of hadronnucleon $(h N)$ interactions near threshold $[1,2]$. Hadronic atom data consist primarily of strong interaction level shifts, widths and yields, derived from $h^{-}$-atom X-ray transitions. These data are analyzed in terms of optical potentials $V_{\mathrm{opt}}^{h}=t_{h N}(\rho) \rho$ which are functionals of the nuclear density $\rho(r)$, capable of handling large data sets across the periodic table in order to identify characteristic entities that may provide a link between experiment and microscopic approaches. Here, $t_{h N}(\rho)$ is a density dependent in-medium $h N t$ matrix at threshold, satisfying the lowdensity limit $t_{h N}(\rho) \rightarrow t_{h N}^{\text {free }}$, with the free-space $t$ matrix $t_{h N}^{\text {free }}$, as $\rho \rightarrow 0$. A schematic summary of lessons gained by analyzing the available hadronic atom data in terms of optical potentials is given in Table 1, where the number of data points included in these analyses is shown in parentheses in the last column. A more exhaustive discussion of $\pi^{-}, \Sigma^{-}$and $K^{-}$atoms follows below.

Table 1. Hadronic atom scenarios which except for $\bar{p}$ atoms are discussed in the present review.

\begin{tabular}{|c|c|c|c|}
\hline hadron & $\operatorname{Re} V_{\mathrm{opt}}$ & $\operatorname{Im} V_{\mathrm{opt}}$ & comments \\
\hline$\pi^{-}$ & $\begin{array}{l}\text { repulsive in bulk } \\
\text { attractive on surface }\end{array}$ & moderate & $\begin{array}{l}\text { excellent data }(100) \\
\text { well understood }\end{array}$ \\
\hline$\Sigma^{-}$ & $\begin{array}{l}\text { repulsive in bulk } \\
\text { attractive outside }\end{array}$ & moderate & $\begin{array}{l}\text { limited data }(23) \\
\text { poorly understood }\end{array}$ \\
\hline$K^{-}$ & $\begin{array}{l}\text { attractive } \\
\text { deep or shallow? }\end{array}$ & absorptive & $\begin{array}{l}\text { good data }(65) \\
\text { open problems }\end{array}$ \\
\hline $\bar{p}$ & model dependent & very absorptive & excellent data $(90)$ \\
\hline
\end{tabular}




\section{Partial restoration of chiral symmetry from pionic atoms}
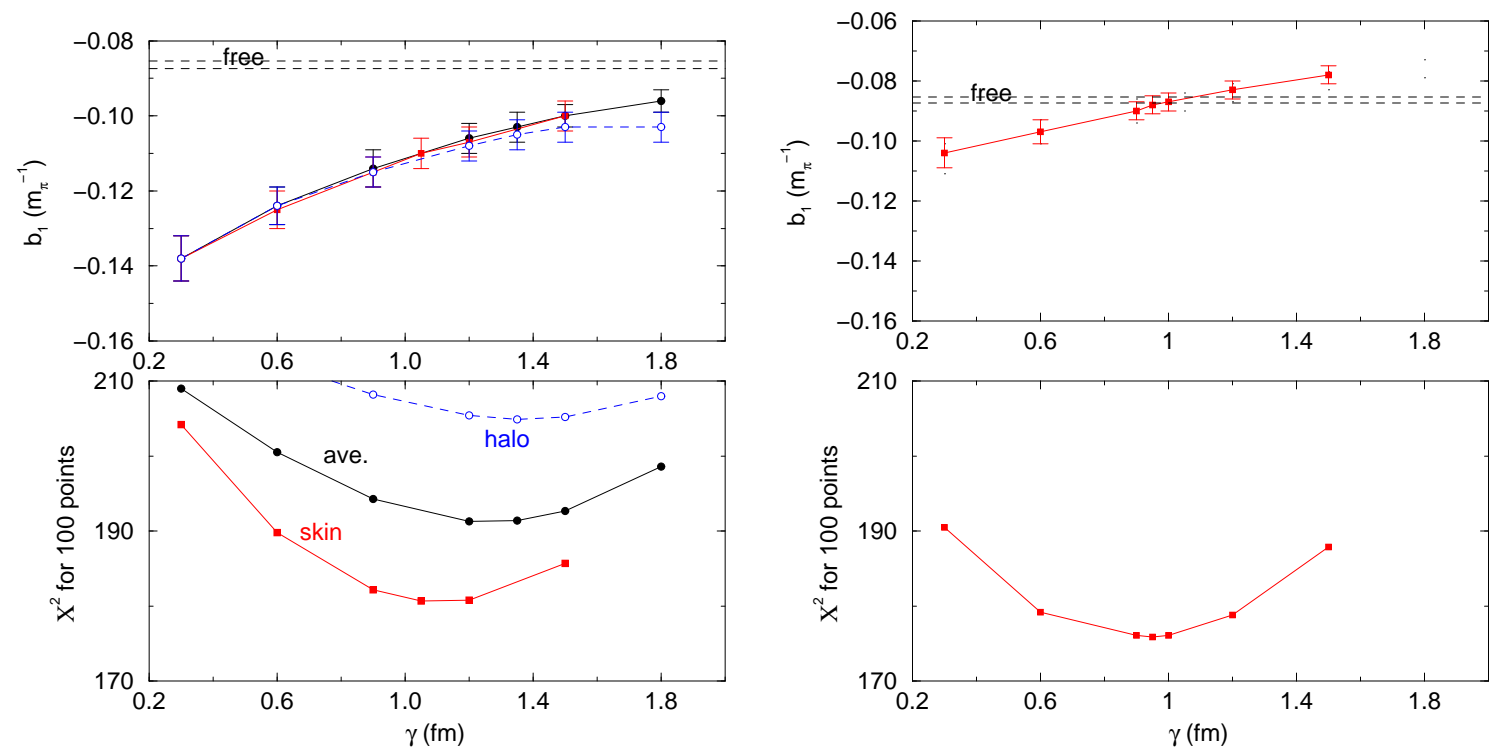

Figure 1. Fits to $100 \pi^{-}$-atom data points from Ne to $\mathrm{U}$ as a function of $\gamma$. Left: densityindependent (DI) $b_{1}$. Right: density-dependent (DD) $b_{1}$. Figure adapted from Ref. [2].

Density independent optical potential global fits to pionic atom data across the periodic table reveal an anomalous $s$-wave repulsion $[1,2]$, a major component of which is due to a too repulsive isovector $\pi N$ amplitude $b_{1}$ with respect to the free-space value $b_{1}^{\text {free }}$. This is demonstrated on the l.h.s. of Fig. 1 which shows results of global fits to pionic atom data as a function of a parameter $\gamma$, related to the difference between neutron and proton rms radii:

$$
r_{n}-r_{p}=\gamma \frac{N-Z}{A}+\delta .
$$

Applying a finite-range folding with rms radius $0.9 \mathrm{fm}$ to the $p$-wave part of $V_{\mathrm{opt}}^{\pi}$, the resulting $\chi^{2}$ minimum for a 'skin' neutron distribution is obtained at $\gamma=1.1 \pm 0.1 \mathrm{fm}$. ${ }^{1}$ The r.h.s. of the figure shows results of global fits with empirical energy dependence imposed on the $s$-wave amplitudes $b_{0}$ and $b_{1}$ [4], and more importantly with a DD renormalization of $b_{1}$ :

$$
b_{1}(\rho)=\frac{b_{1}}{1-\frac{\sigma \rho}{m_{\pi}^{2} f_{\pi}^{2}}},
$$

where $f_{\pi}=92.4 \mathrm{MeV}$ is the pion weak decay constant and $\sigma \approx 50 \mathrm{MeV}$ is the $\pi N \sigma$ term. Eq. (2) was derived by Weise [5] considering an in-medium extension of the Tomozawa-Weinberg (TW) LO chiral limit for $b_{1}$ in terms of $f_{\pi}[6]$ which is then related to the quark condensate $<\bar{q} q>$ :

$$
b_{1}(\rho)=-\frac{\mu_{\pi N}}{8 \pi f_{\pi}^{2}(\rho)}, \quad \frac{f_{\pi}^{2}(\rho)}{f_{\pi}^{2}}=\frac{<\bar{q} q>_{\rho}}{<\bar{q} q>_{0}} \simeq 1-\frac{\sigma \rho}{m_{\pi}^{2} f_{\pi}^{2}} .
$$

The figure makes it evident that the magnitude of $b_{1}$ on the r.h.s., following the DD renormalization, is systematically smaller than that on the l.h.s., and at the $\chi^{2}$ minimum it agrees perfectly with $b_{1}^{\text {free }}$.

1 For a recent discussion of the role of neutron distributions in hadronic atoms, see Ref. [3]. 
Table 2. Values of $b_{1}$ derived from density-independent fits to pionic atom data sets listed in Ref. [9]. For comparison, $b_{1}^{\text {free }}=-0.0864 \pm 0.0010 m_{\pi}^{-1}$ [10]. 'Deep' refers to $1 s \pi^{-}$'deeply bound' states in ${ }^{205} \mathrm{~Pb}[11]$ and ${ }^{115,119,123} \mathrm{Sn}[8]$ observed in $\left(\mathrm{d},{ }^{3} \mathrm{He}\right)$ experiments at GSI.

\begin{tabular}{lccc}
\hline data & $\begin{array}{c}\text { global } \\
{ }^{20} \mathrm{Ne} \text { to }{ }^{238} \mathrm{U}\end{array}$ & $\begin{array}{c}\text { light } N=Z \\
+ \text { 'deep' } 1 s \text { only }\end{array}$ & $\begin{array}{c}\text { 'deep' } \\
1 s \text { only }\end{array}$ \\
\hline points & 100 & 20 & 8 \\
$b_{1}\left(m_{\pi}^{-1}\right)$ & $-0.104 \pm 0.006$ & $-0.104 \pm 0.013$ & $-0.130 \pm 0.036$ \\
\hline
\end{tabular}

A similar conclusion was reached in Refs. [7, 8] from measurements of $1 s$ 'deeply bound' pionic atoms of Sn isotopes. The advantage of using $1 s$ 'deeply bound' levels is that the $p$-wave $\pi N$ interaction plays there a secondary role, but this merit is more than compensated by the considearably increased errors associated with smaller data sets, as shown in Table 2. The uncertainty listed in the fourth column makes it clear that the 'deeply bound' atoms alone do not give conclusive evidence for the need to renormalize $b_{1}$. In fact, Suzuki et al. [8] considered 'deeply bound' $1 s$ levels in three Sn isotopes together with 'normal' $1 s$ levels in ${ }^{16} \mathrm{O},{ }^{20} \mathrm{Ne}$ and ${ }^{28} \mathrm{Si}, 12$ data points in total yielding $b_{1}=-0.1149 \pm 0.0074 m_{\pi}^{-1}$. However, this small uncertainty excludes the uncertainty from the $p$-wave $\pi N$ potential which was held fixed in their analysis. A more realistic uncertainty for this type of deduction is given in column 3 of the table.
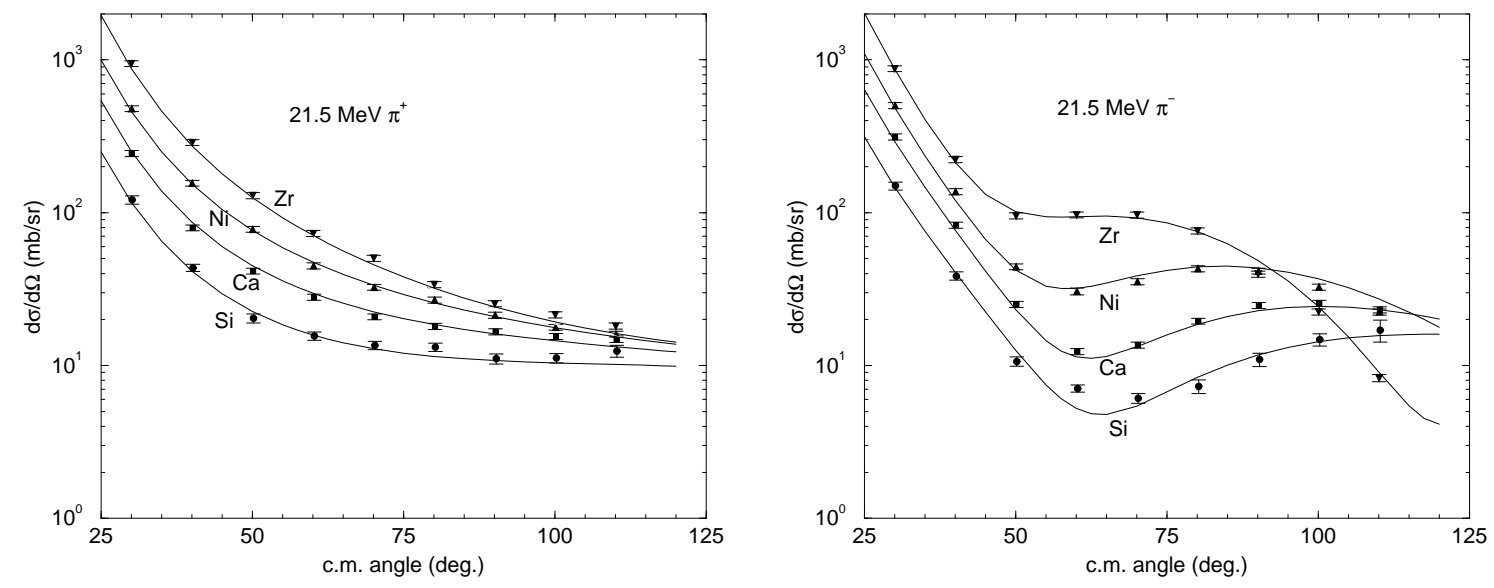

Figure 2. Low energy $\pi^{ \pm}$nucleus elastic scattering angular distributions [12] reproduced with $b_{1}(\rho)$ ansatz, Eq. (2).

The renormalization of $b_{1}$ derived from pionic atoms is consistent with that shown recently to be also required in low energy $\pi$-nucleus scattering, as demonstrated in Fig. 2 for $21.5 \mathrm{MeV}$ $\pi^{ \pm}$scattered off isotopes of $\mathrm{Si}, \mathrm{Ca}, \mathrm{Ni}$ and $\mathrm{Zr}$ at PSI [12].

\section{3. $\Sigma$ Nuclear repulsion from $\Sigma^{-}$atoms}

A vast body of $\left(K^{-}, \pi^{ \pm}\right)$spectra indicate a repulsive and moderately absorptive $\Sigma$ nuclear potential $V^{\Sigma}$, with a substantial isospin dependence $[13,14]$. These data, including recent $\left(\pi^{-}, K^{+}\right)$spectra [15] and related DWIA analyses [16], provide credible evidence that $\Sigma$ hyperons generally do not bind in nuclei. A repulsive component of a DD $\Sigma$ nuclear potential was already 

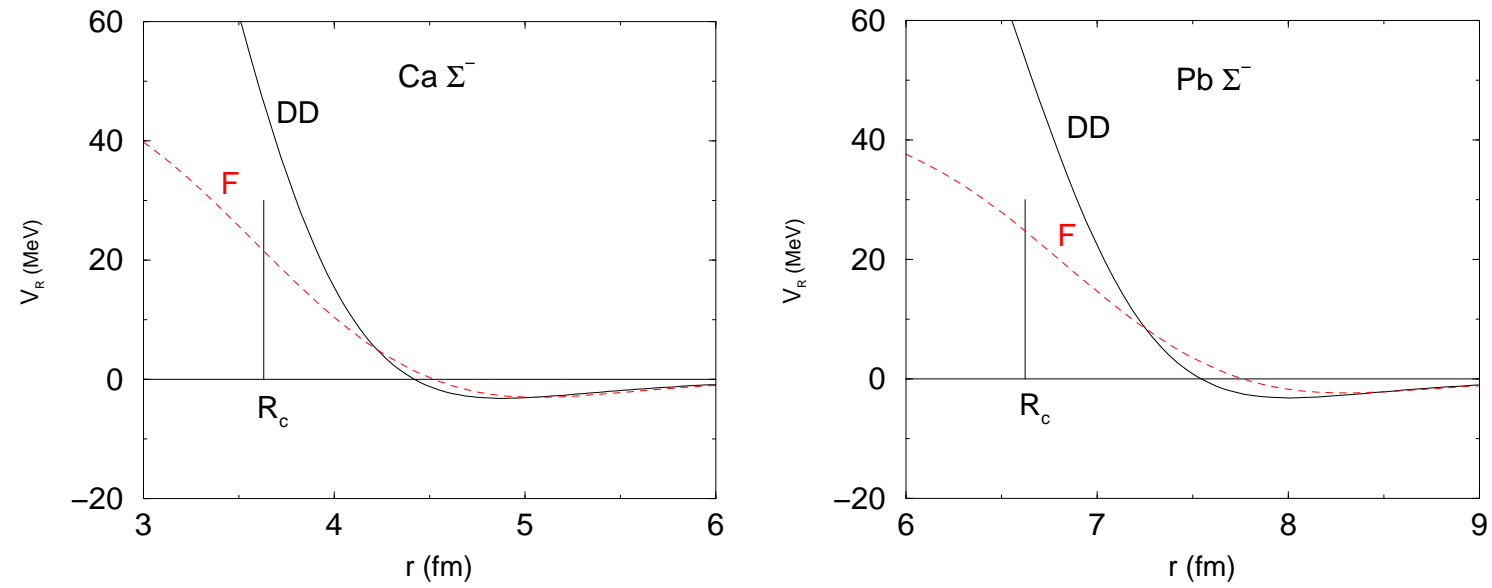

Figure 3. $V_{\mathrm{R}}^{\Sigma}$ from fits to $\Sigma^{-}$atoms [2]. The transition from attraction to repulsion occurs well outside of $R_{c}$, the half-density radius of the charge distribution in $\mathrm{Ca}$ (1.h.s.) and $\mathrm{Pb}$ (r.h.s.).

deduced in the mid 1990s from $\Sigma^{-}$atom data $[17,18]$, as shown in Fig. 3. In fact, $V_{\mathrm{R}}^{\Sigma}$ is attractive at low densities outside the nucleus, as enforced by the observed 'attractive' $\Sigma^{-}$ atomic level shifts, changing into repulsion on approach of the nuclear radius. The precise magnitude and shape of $V_{\mathrm{R}}^{\Sigma}$ within the nucleus, however, are model dependent as demonstrated by the difference between potentials DD and F (defined in the Appendix). This repulsion bears interesting consequences for the balance of strangeness in the inner crust of neutron stars, primarily by delaying to higher densities, or even aborting the appearance of $\Sigma^{-}$hyperons, as shown in Fig. 4.
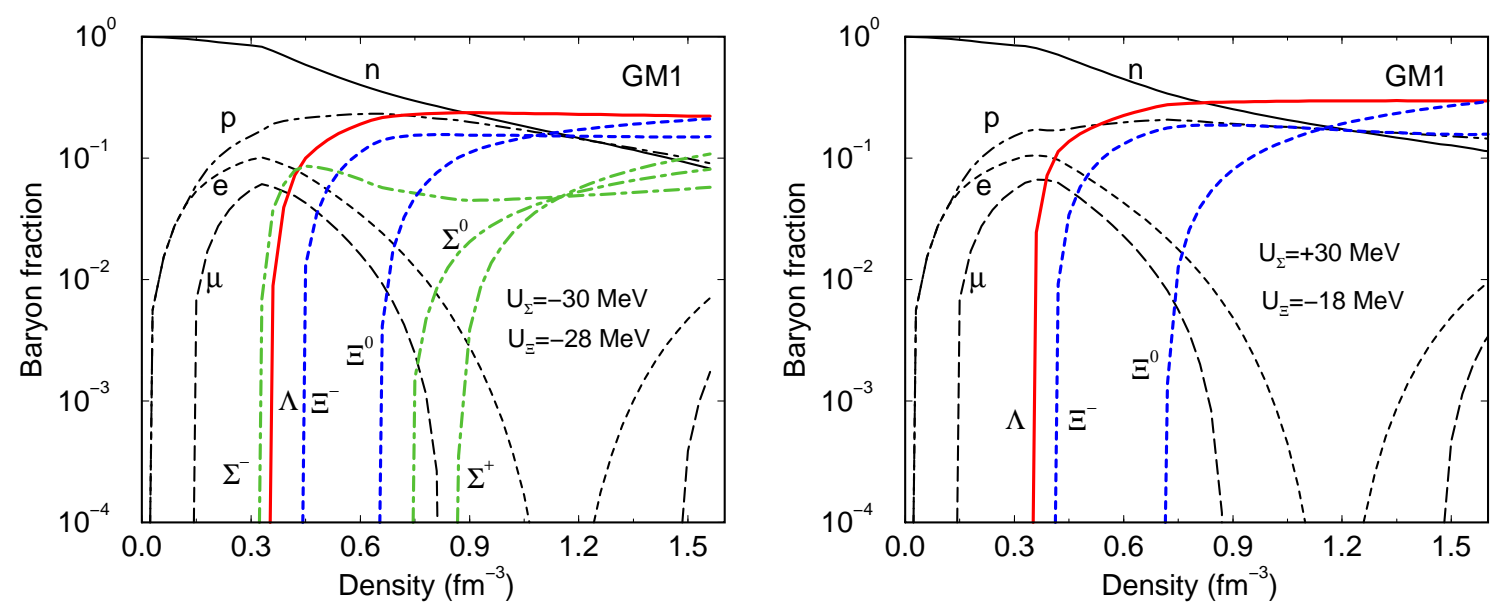

Figure 4. RMF calculations of baryon \& lepton fractions in neutron star matter for different scenarios of hyperon nuclear potential depths $U_{Y}$. Figure adapted from Ref. [19].

The $G$-matrices constructed from Nijmegen soft-core potential models have progressed throughout the years to produce $\Sigma$ repulsion in symmetric nuclear matter, as demonstrated in Table 3 using the parametrization

$$
V_{R}^{\Sigma}=V_{0}^{\Sigma}+\frac{1}{A} V_{1}^{\Sigma} \mathbf{T}_{A} \cdot \mathbf{t}_{\Sigma}
$$


Table 3. Isoscalar and isovector $\Sigma$ nucleus potentials, Eq. (4) in MeV, calculated in Nijmegen soft-core potential models [20] at $k_{F}=1.35 \mathrm{fm}^{-1}$, corresponding to nuclear-matter density.

\begin{tabular}{cccccccc}
\hline & $97 \mathrm{f}$ & $04 \mathrm{~d}$ & $06 \mathrm{~d}$ & $08 \mathrm{a}$ & $08 \mathrm{~b}$ & phenom. & Ref. \\
\hline$V_{0}^{\Sigma}$ & -13.9 & -26.0 & -1.2 & +13.4 & +20.3 & $+30 \pm 20$ & {$[2,16]$} \\
$V_{1}^{\Sigma}$ & -30.4 & +30.4 & +52.6 & +64.5 & +85.2 & $\approx+80$ & {$[21]$} \\
\hline
\end{tabular}

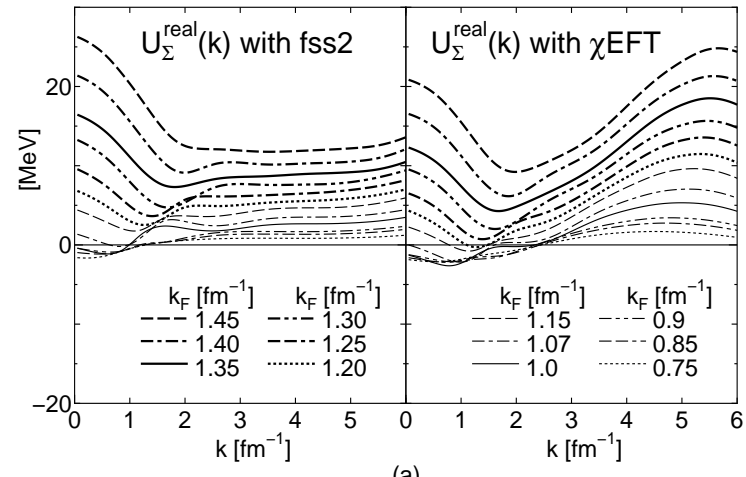

(a)

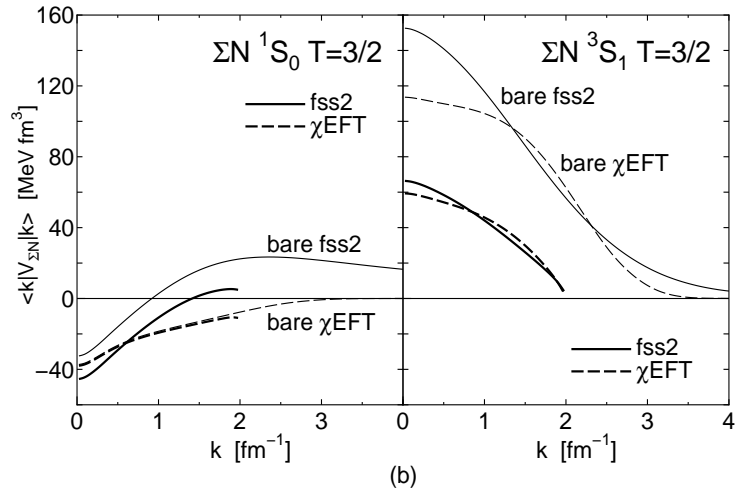

(b)

Figure 5. Left: Isoscalar $\Sigma$ nuclear potentials calculated in two models, Refs. [22, 23]. Right: $(\Sigma N)_{T=3 / 2}$ potentials calculated in these models. Figure adapted from Ref. [24].

In the latest Nijmegen ESC08 model [20], this repulsion is dominated by repulsion in the $T=3 / 2,{ }^{3} S_{1}-{ }^{3} D_{1} \Sigma N$ channel where a strong short distance Pauli exclusion repulsion for quarks arises in SU(6) quark-model RGM [22] and in chiral EFT [23] calculations, as seen on the r.h.s. of Fig. 5. These model calculations also lead to $\Sigma$ nuclear repulsion, shown in momentum space on the l.h.s. of the figure. A strong repulsion appears also in a recent $\mathrm{SU}(3)$ chiral perturbation calculation [25] which yields $V_{0}^{\Sigma} \approx 60 \mathrm{MeV}$. Phenomenologically $V_{0}^{\Sigma}>0$ and $V_{1}^{\Sigma}>0$, as listed in the table, and the resulting $\Sigma$-nuclear potential $V_{R}^{\Sigma}$ is repulsive. ${ }^{2}$

\section{4. $\bar{K}$-nucleus potentials from $K^{-}$atoms}

The gross features of low-energy $\bar{K} N$ physics are encapsulated in the leading-order TomozawaWeinberg (TW) vector term of the chiral effective Lagrangian [6]. The Born approximation for the $\bar{K}$-nuclear potential $V_{\mathrm{TW}}^{\bar{K}}$ due to this TW interaction term yields a sizable attraction:

$$
V_{\mathrm{TW}}^{\bar{K}}=-\frac{3}{8 f_{\pi}^{2}} \rho \sim-55 \frac{\rho}{\rho_{0}} \quad(\mathrm{MeV})
$$

for $\rho_{0}=0.16 \mathrm{fm}^{-3}$. Iterating the TW term plus the less significant NLO terms, within an in-medium coupled-channel approach constrained by the $\bar{K} N-\pi \Sigma-\pi \Lambda$ data near the $\bar{K} N$ threshold, roughly doubles this $\bar{K}$-nucleus attraction [29]. A major uncertainty in these chirally based studies arises from fitting the $\Lambda(1405)$ resonance by the imaginary part of the $(\pi \Sigma)_{I=0}$

2 In the case of ${ }_{\Sigma}^{4} \mathrm{He}$, the only known quasibound $\Sigma$ hypernucleus [26, 27], the isovector term provides substantial attraction owing to the small value of $A$ towards binding the $T=1 / 2$ hypernuclear configuration, while the isoscalar repulsion reduces the quasibound level width [28]. 

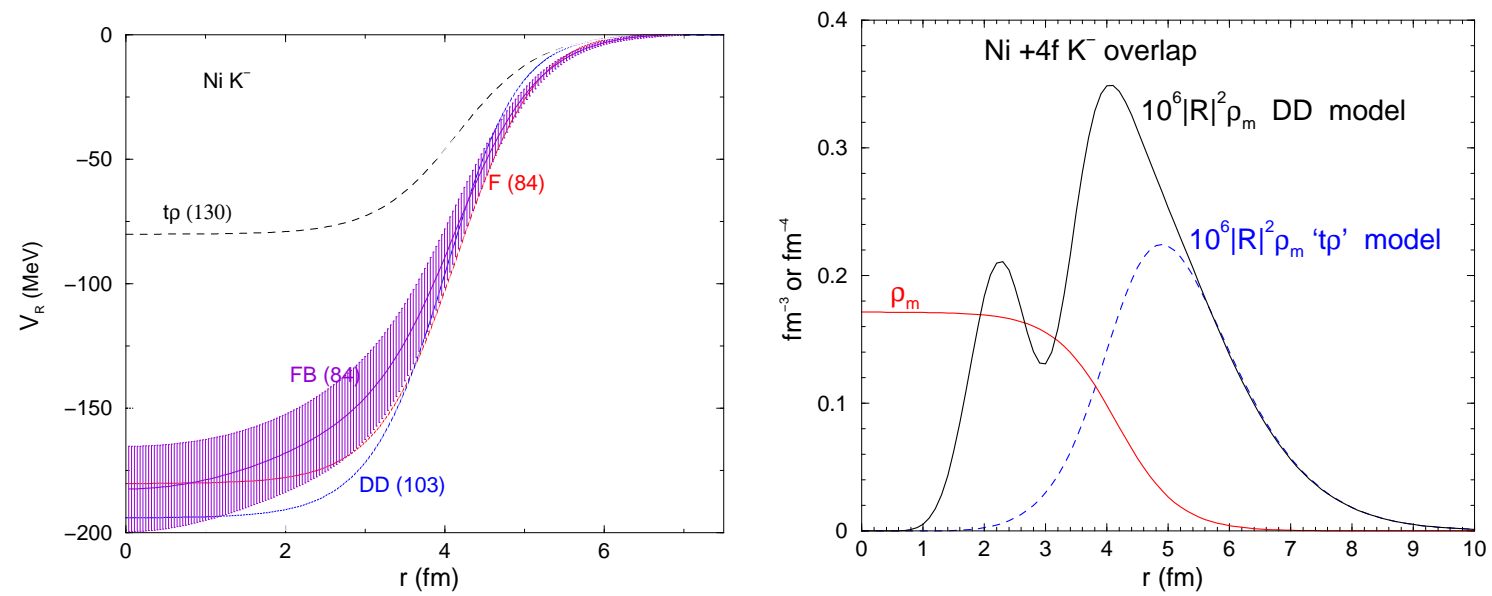

Figure 6. Left: $V_{R}^{\bar{K}}$ in Ni from global fits to $65 K^{-}$atom data points, with $\chi^{2}$ values in parentheses [2]. The shaded area approximates the uncertainty in the Fourier-Bessel fit. Right: Overlap of $K^{-}$atomic $4 f$ radial wavefunctions $R$ squared with Ni matter density $\rho_{m}$.

amplitude calculated within the same coupled channel chiral scheme. Yet, irrespective of this uncertainty, the $\Lambda(1405)$ which may be viewed as a $K^{-} p$ quasibound state quickly dissolves in the nuclear medium at low density, so that the repulsive free-space scattering length $a_{K^{-}}$, as function of $\rho$, becomes attractive well below $\rho_{0}$. Adding the weakly density dependent $I=1$ attractive scattering length $a_{K^{-} n}$, the resulting in-medium $\bar{K} N$ isoscalar scattering length $b_{0}(\rho)=\frac{1}{2}\left(a_{K^{-} p}(\rho)+a_{K^{-} n}(\rho)\right)$ translates into a strongly attractive $V^{\bar{K}}[30,31]$ :

$$
V_{R}^{\bar{K}}(\rho) \sim-\frac{2 \pi}{\mu_{K N}} \operatorname{Re} b_{0}\left(\rho_{0}\right) \rho_{0} \frac{\rho}{\rho_{0}} \approx-110 \frac{\rho}{\rho_{0}} \quad(\mathrm{MeV}) .
$$

Shallower potentials, $V_{R}^{\bar{K}}\left(\rho_{0}\right) \sim-(40-60) \mathrm{MeV}$, were obtained by imposing a Watsonlike self-consistency requirement [30, 32]. It turns out, however, that stronger attraction, $V_{R}^{\bar{K}}\left(\rho_{0}\right) \sim-(80-90) \mathrm{MeV}$, arises in similar chiral approaches [33] when imposing the same requirement while considering the energy dependence of the in-medium $\bar{K} N$ scattering amplitude below threshold [34].

Comprehensive fits to the strong-interaction shifts and widths of $K^{-}$-atom levels, begun in the mid 1990s [35], have yielded DD deeply attractive and strongly absorptive optical potentials with nuclear-matter depth $-V_{R}^{\bar{K}}\left(\rho_{0}\right) \sim(150-200) \mathrm{MeV}$ at threshold [35]. The 1.h.s. of Fig. 6 illustrates for ${ }^{58} \mathrm{Ni}$ the real part of $\bar{K}$-nucleus potentials obtained from a global fit to the data in several models and, in parentheses, the corresponding values of $\chi^{2}$ for $65 K^{-}$-atom data points. A model-independent Fourier-Bessel (FB) fit [36] is also shown, within an error band. Just three terms in the FB series, added to a $t \rho$ potential, suffice to achieve a $\chi^{2}$ as low as 84 and to make the potential extremely deep, in agreement with the density-dependent best-fit potentials $\mathrm{DD}$ and $\mathrm{F}$. In particular, potential $\mathrm{F}$ provides by far the best fit ever reported for any global $K^{-}$-atom data fit [37], and the lowest $\chi^{2}$ value as reached by the FB method.

Shown on the r.h.s. of Fig. 6 are overlaps of the $4 f$ atomic radial wavefunction squared with the matter density $\rho_{m}$ in ${ }^{58} \mathrm{Ni}$ for two of the models exhibited on the l.h.s. of the figure. The $4 f$ atomic orbit is the last circular $K^{-}$atomic orbit from which the $K^{-}$meson undergoes nuclear absorption. The figure demonstrates that, whereas this overlap for the shallower $t \rho$ potential peaks at nuclear density of order $10 \%$ of $\rho_{0}$, it peaks at about $60 \%$ of $\rho_{0}$ for the deeper DD potential and has a secondary peak well inside the nucleus. The double-peak structure indicates 
Table 4. Full and reduced $K^{-}$atom data set fits. The reduced set consists of $2 p, 3 d, 4 f, 5 g, 7 i$ shifts, widths and yields in $\mathrm{C}, \mathrm{Si}, \mathrm{Ni}, \mathrm{Sn}$ and $\mathrm{Pb}$ targets, respectively [39].

\begin{tabular}{lllllll}
\hline & \multicolumn{3}{c}{ shallow potential } & \multicolumn{3}{c}{ deep potential } \\
$\mathrm{N}$ & $\operatorname{Re} b\left(\rho_{0}\right)$ & $\operatorname{Im} b\left(\rho_{0}\right)$ & $\chi^{2}$ & $\operatorname{Re} b\left(\rho_{0}\right)$ & $\operatorname{Im} b\left(\rho_{0}\right)$ & $\chi^{2}$ \\
\hline 65 & $0.62 \pm 0.05$ & $0.93 \pm 0.04$ & 130 & $1.44 \pm 0.03$ & $0.59 \pm 0.03$ & 84 \\
15 & $0.78 \pm 0.13$ & $0.92 \pm 0.11$ & 44 & $1.47 \pm 0.05$ & $0.55 \pm 0.06$ & 26 \\
\hline
\end{tabular}

the existence of a $K^{-}$strong-interaction $\ell=3$ quasibound state for the DD potential. It is clear that whereas within the $t \rho$ potential there is no sensitivity to the interior of the nucleus, the opposite holds for the density dependent $\mathrm{F}$ potential which accesses regions of full nuclear density. This owes partly to the smaller imaginary part of F.

Given the repercussions of deeply attractive potentials on the equation of state of dense matter, it is important to explore the stability of these best-fit solutions to variations in the data selection and the fitting procedure. The most obvious question to ask is whether the resulting best-fit potentials depend strongly on the size, composition and accuracy of the data set studied. Regarding size and composition, following an earlier discussion [38] it has been observed recently [39] that the DD deep potentials and the DI relatively shallow potentials, as well as the superiority of DD to DI in terms of quality of fit, persist upon decreasing the size of the data set. This is demonstrated in Table 4 upon reducing the 65 data point global set down to 15 data points from five targets spread over the entire periodic table ( $\mathrm{C}, \mathrm{Si}, \mathrm{Ni}, \mathrm{Sn}, \mathrm{Pb}$ ). Similar results hold for any four out of these five targets. It makes sense then to repeat some of the $30-40$ years old $K^{-}$atom measurements, making use of modern techniques, in order to acquire a minimum size canonical set of data with reduced statistical errors and with common systematics. For the specific set proposed in Ref. [39], the (lower level) widths are directly measurable yet not excessively large to make it difficult to observe the feeding X-ray transition above the background. Similarly, the relative yields of the upper to lower level transitions are of the order of $10 \%$ and higher. Fitting to such a data set with improved accuracy could resolve the issue of deep vs. shallow potentials and determine how deep is 'deep'.

A fairly new and independent evidence in favor of extremely deep $\bar{K}$-nucleus potentials is provided by $\left(K^{-}, n\right)$ and $\left(K^{-}, p\right)$ spectra taken at KEK on ${ }^{12} \mathrm{C}[40]$ and very recently also on ${ }^{16} \mathrm{O}$ [41] at $p_{K^{-}}=1 \mathrm{GeV} / \mathrm{c}$. The ${ }^{12} \mathrm{C}$ spectra are shown on the 1.h.s. of Fig. 7 , where the solid lines represent calculations (outlined in Ref. [43]) using potential depths in the range $160-190 \mathrm{MeV}$. The dashed lines correspond to using relatively shallow potentials of depth about $60 \mathrm{MeV}$ which may be considered excluded by these data. However, Magas et al. [44] have recently expressed concerns about protons of reactions other than those directly emanating in the $\left(K^{-}, p\right)$ reaction and which could explain part of the bound-state region of the measured spectrum without invoking a very deep $\bar{K}$-nuclear potential. A sufficientlly deep potential would allow quasibound states bound by over $100 \mathrm{MeV}$, for which the major $\bar{K} N \rightarrow \pi \Sigma$ decay channel is blocked, resulting in relatively narrow $\bar{K}$-nuclear states. Of course, a fairly sizable extrapolation is involved in this case using an energy-independent potential determined largely near threshold. Furthermore, the best-fit $V_{I}^{\bar{K}}$ imaginary depths of $40-50 \mathrm{MeV}$ imply that $\bar{K}$-nuclear quasibound states are broad, as studied in Refs. [37, 47].

A robust consequence of the sizable $\bar{K}$-nucleus attraction is that $K^{-}$condensation, when hyperon degrees of freedom are ignored, could occur in neutron star matter at about 3 times nuclear matter density, as shown on the r.h.s. of Fig. 7. Comparing it with Fig. 4 for neutron stars, but where strangeness materialized through hyperons, one may ask whether $\bar{K}$ mesons 

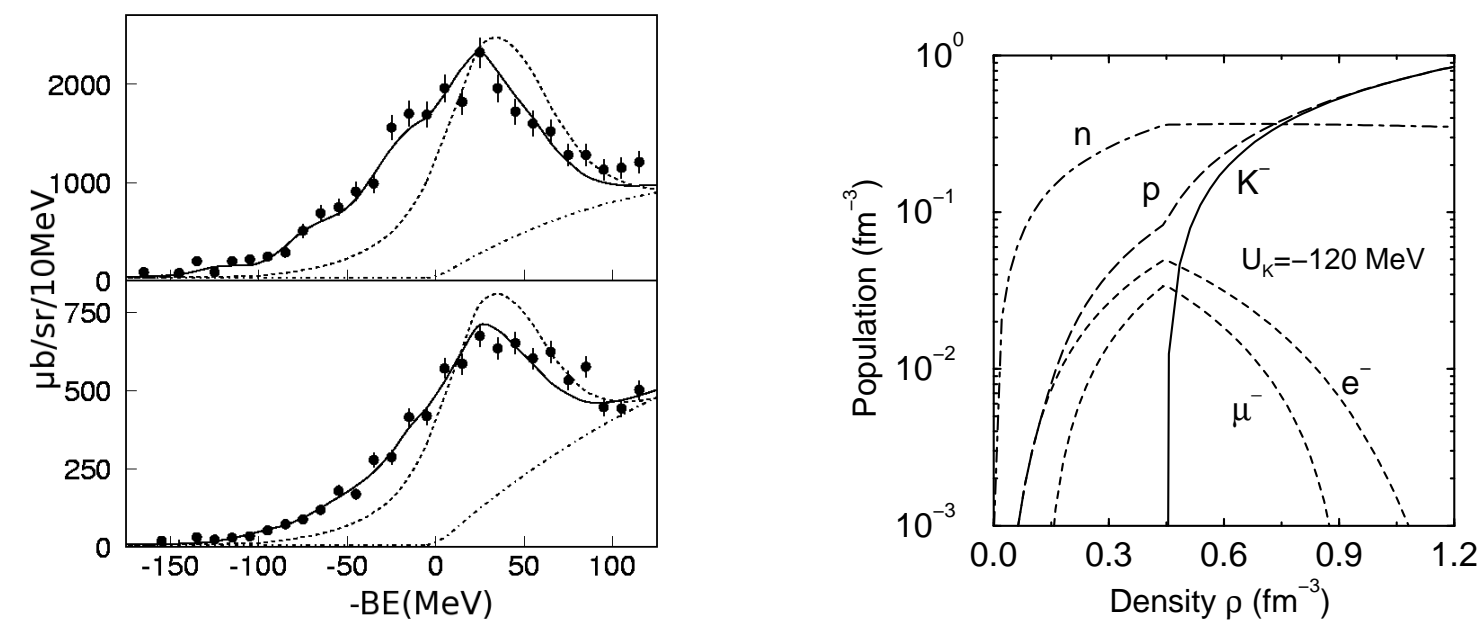

Figure 7. Left: KEK-PS E548 missing mass $\left(K^{-}, n\right)$ (upper) \& $\left(K^{-}, p\right)$ (lower) spectra on ${ }^{12} \mathrm{C}$ at $p_{K^{-}}=1 \mathrm{GeV} / \mathrm{c}$ [40]. Right: calculated neutron-star population as a function of density. The neutron density stays nearly constant once kaons condense [42].
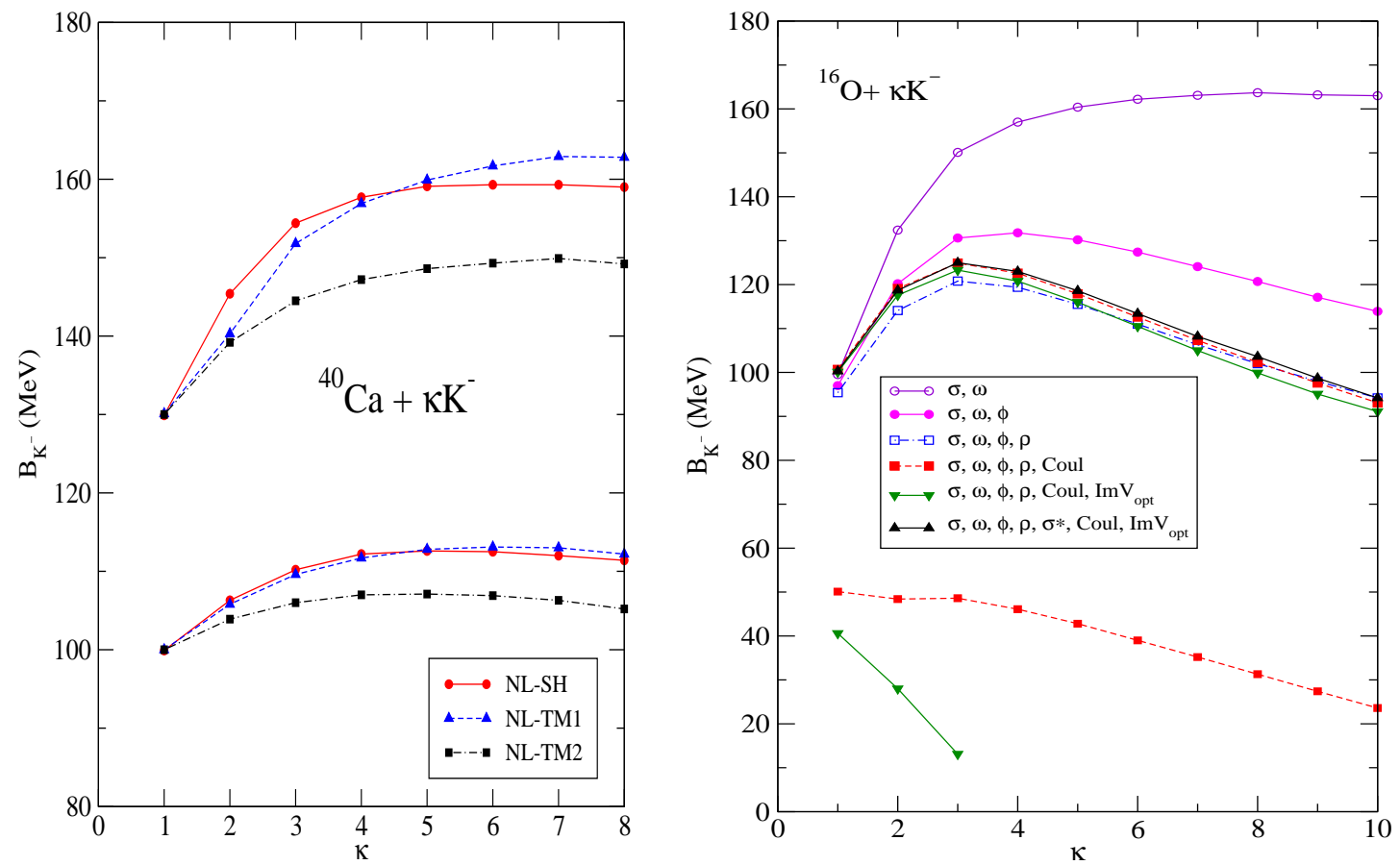

Figure 8. Saturation of $K^{-}$separation energy $B_{\bar{K}}$ in RMF calculations of multi $K^{-}$nuclei as a function of the number $\kappa$ of $K^{-}$mesons [48]. Left: RMF model dependence. Right: dependence on RMF ingredients.

condense also in the presence of hyperons. This question was posed within RMF calculations of neutron star matter long ago and answered negatively $[45,46]$, but only recently it was posed 
for strange hadronic matter in Ref. [48] by calculating multi- $\bar{K}$ nuclear configurations. Fig. 8 demonstrates a remarkable saturation of $K^{-}$separation energies $B_{K^{-}}$calculated in multi- $K^{-}$ nuclei, independently of the applied RMF model as shown on the l.h.s. for three different nuclear RMF schemes. The r.h.s. of the figure demonstrates that this saturation persists already in the most straightforward $\sigma+\omega$ model, primarily owing to the repulsion induced by the vector $\omega$ field between like $\bar{K}$ mesons. The additional vector fields $\rho$ and $\phi$ only add repulsion, thus strengthening the saturation. The effect of $V_{I}^{\bar{K}}$ is noticeable only below $B_{\bar{K}} \approx 100 \mathrm{MeV}$, as seen by the departure of the lowest green line with respect to the lowest red line.

The saturation values of $B_{K^{-}}$do not allow conversion of hyperons to $\bar{K}$ mesons through the strong decays $\Lambda \rightarrow p+K^{-}$or $\Xi^{-} \rightarrow \Lambda+K^{-}$in multi-strange hypernuclei, which therefore remain the lowest-energy configuration for multi-strange systems [49]. This provides a powerful argument against $\bar{K}$ condensation in the laboratory, under strong-interaction equilibrium conditions [48]. It does not apply to kaon condensation in neutron stars, where equilibrium configurations are determined by weak-interaction conditions. This work has been recently generalized to multi- $K^{-}$hypernuclei [50].

\section{Appendix: density dependent optical potentials}

Here we specify the functional form of two density dependent optical potentials used in studies of hadronic atoms. For a recent application to $K^{-}$atoms, see Table 1 of Ref. [37].

- The DD form is based on modifying the effective scattering length $b_{0}$ (e.g. Eq. (6)):

$$
V^{h}(r) \sim-\frac{2 \pi}{\mu_{h N}} b_{0} \rho(r) \Rightarrow b_{0} \rightarrow b_{0}+B_{0}\left\{\frac{\rho(r)}{\rho_{0}}\right\}^{\alpha}, \alpha>0
$$

where $\rho_{0}=0.16 \mathrm{fm}^{-3}$ is a central nuclear density. It is possible then to respect the 'low density limit' by keeping $b_{0}$ fixed, $b_{0}=b_{0}^{\text {free }}$, while varying the parameters $B_{0}$ and $\alpha$.

- The $\mathrm{F}$ form is based on modifying $b_{0}$ as follows:

$$
b_{0} \rightarrow B_{0} F(r)+b_{0}[1-F(r)] .
$$

The density-like function $F(r)$ is defined as

$$
F(r)=\frac{1}{e^{x}+1}, \quad x=\frac{r-R_{x}}{a_{x}} .
$$

Clearly, $F(r) \rightarrow 1$ for $r<<R_{x}$ which defines an internal region and similarly $[1-F(r)] \rightarrow 1$ for $r>>R_{x}$ which defines an external region. Thus $R_{x}$ forms an approximate border between internal and external regions, and if $R_{x}$ is close to the nuclear surface then the two regions do correspond to the high-density and low-density regions of nuclei, respectively. In global fits across the periodic table, $R_{x}$ is parametrized as $R_{x}=R_{x 0} A^{1 / 3}+\delta_{x}$ and the parameters $B_{0}, R_{x 0}$ and $\delta_{x}$ are varied upon in the least-squares fit, while gridding on values of $a_{x}$ around $0.5 \mathrm{fm}$. The parameter $b_{0}$ may be held fixed at its free $h N$ value, but the results often depend very little on its precise value.

\section{Acknowledgments}

On the occasion of Gerry Brown's 85th birthday Festschrift, we dedicate this mini review to him who commissioned our two past reviews $[1,2]$ on similar subjects. This work was supported in part by the SPHERE collaboration within the HadronPhysics2 Project No. 227431 of the EU initiative FP7. 


\section{References}

[1] Batty C J, Friedman E and Gal A 1997 Phys. Rept. 287385

[2] Friedman E and Gal A 2007 Phys. Rept. 452 89, and references therein

[3] Friedman E 2009 Hyp. Int. 193 33, and references therein

[4] Friedman E and Gal A 2004 Phys. Lett. B 57885

[5] Weise W 2001 Nucl. Phys. A 690 98c

[6] Tomozawa Y 1966 Nuovo Cimento A 46 707, Weinberg S 1966 Phys. Rev. Lett. 17616

[7] Kolomeitsev E E, Kaiser N and Weise W 2003 Phys. Rev. Lett. 90092501

[8] Suzuki K et al. 2004 Phys. Rev. Lett. 92072302

[9] Friedman E and Gal A 2003 Nucl. Phys. A 724143

[10] Marton J 2007 Nucl. Phys. A 790 328c

[11] Geissel H et al. 2002 Phys. Rev. Lett. 88122301

[12] Friedman E et al. 2004 Phys. Rev. Lett. 93 122302, 2005 Phys. Rev. C 72034609

[13] Dover C B, Millener D J and Gal A 1989 Phys. Rept. 184 1, and references therein

[14] Bart S et al. [BNL E887] 1999 Phys. Rev. Lett. 835238

[15] Noumi H et al. [KEK E438] 2002 Phys. Rev. Lett. 89 072301, 2003 Phys. Rev. Lett. 90 049902(E), Saha P K et al. 2004 Phys. Rev. C 70044613

[16] Kohno M, Fujiwara Y, Watanabe Y, Ogata K and Kawai M 2004 Prog. Theor. Phys. 112895 , 2006 Phys. Rev. C 74 064613, Harada T and Hirabayashi Y 2005 Nucl. Phys. A 759143,2006767206

[17] Batty C J, Friedman E and Gal A 1994 Phys. Lett. B 335 273, Prog. Theor. Phys. Suppl. 117227

[18] Mareš J, Friedman E, Gal A and Jennings B K 1995 Nucl. Phys. A 594311

[19] Schaffner-Bielich J 2010 Nucl. Phys. A 835 279, and references therein

[20] Rijken Th A, Nagels M M and Yamamoto Y 2010 Nucl. Phys. A 835 160, and references therein

[21] Dover C B, Gal A and Millener D J 1984 Phys. Lett. B 138337

[22] Fujiwara Y, Suzuki Y and Nakamoto C 2007 Prog. Part. Nucl. Phys. 58 439, and references therein

[23] Polinder H, Haidenbauer J and Meißner U G 2006 Nucl. Phys. A 779244

[24] Kohno M 2010 Phys. Rev. C 81014003

[25] Kaiser N 2005 Phys. Rev. C 71068201

[26] Hayano R S et al. 1989 Phys. Lett. B 231355

[27] Nagae T et al. [BNL E905] 1998 Phys. Rev. Lett. 801605

[28] Harada T 1998 Phys. Rev. Lett. 815287

[29] Borasoy B, Nißler R and Weise W 2005 Eur. Phys. J. A 2579

[30] Cieplý A, Friedman E, Gal A and Mareš J 2001 Nucl. Phys. A 696173

[31] Weise W and Härtle R 2008 Nucl. Phys. A 804173

[32] Ramos A and Oset E 2000 Nucl. Phys. A 671481

[33] Cieplý A and Smejkal J 2010 Eur. Phys. J. A 43191

[34] Cieplý A, Friedman E, Gal A, Gazda D and Mareš J 2011 Phys. Lett. B 702 402, see also Cieplý A, Friedman E, Gal A and Krejčiř́ík V 2011 Phys. Lett. B 698226

[35] Friedman E, Gal A and Batty C J 1993 Phys. Lett. B 308 6, 1994 Nucl. Phys. A 579518

[36] Barnea N and Friedman E 2007 Phys. Rev. C 75 022202(R)

[37] Mareš J, Friedman E and Gal A 2006 Nucl. Phys. A 77084

[38] Friedman E, Gal A, Mareš J and Cieplý A 1999 Phys. Rev. C 60024314

[39] Friedman E 2011 Int. J. Mod. Phys. A 26468 (Proc. Int. Conf. on Meson Physics, Krakow 2010)

[40] Kishimoto T 2007 et al. [KEK E548] Prog. Theor. Phys. 118181

[41] Kishimoto T 2009 Nucl. Phys. A 827 321c

[42] Glendenning N K and Schaffner-Bielich J 1999 Phys. Rev. C 60025803

[43] Yamagata J, Nagahiro H and Hirenzaki S 2006 Phys. Rev. C 74014604

[44] Magas V K, Yamagata-Sekihara J, Hirenzaki S, Oset E and Ramos A 2010 Phys. Rev. C 81024609

[45] Knorren R, Prakash M and Ellis P J 1995 Phys. Rev. C 523470

[46] Schaffner J and Mishustin I N 1996 Phys. Rev. C 531416

[47] Gazda D, Friedman E, Gal A and Mareš J 2007 Phys. Rev. C 76055204

[48] Gazda D, Friedman E, Gal A and Mareš J 2008 Phys. Rev. C 77045206

[49] Schaffner-Bielich J and Gal A 2000 Phys. Rev. C 62 034311, and references therein

[50] Gazda D, Friedman E, Gal A and Mareš J 2009 Phys. Rev. C 80035205 